\title{
Model-Based Control of a Nonlinear One Dimensional Magnetic Levitation with a Permanent-Magnet Object
}

\author{
Zhenyu Yang, Gerulf K.M. Pedersen and Jørgen H. Pedersen \\ Esbjerg Institute of Technology, Aalborg University
}

Denmark

\section{Introduction}

The Electromagnet levitation technique has been popularly used in transport and industrial felds in recent decades, such as high-speed levitation trains, frictionless magnetic bearings, and high precision control in semiconductor manufacturing (CST (1996); Kim et al. (1998)). Due to its high efficiency and good environmental orientation, the application of this technique is extensively growing. For instance, the attitude of a small-sized satellite can be efficiently controlled by using the electromagnetic force generated from the interaction between the on- board (controlled) electrical field and the earth magnetic field (Wisniewski \& Stoustrup (2004)).

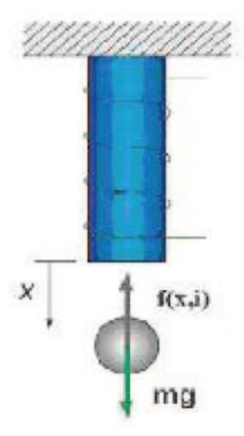

(a) Conventional structure

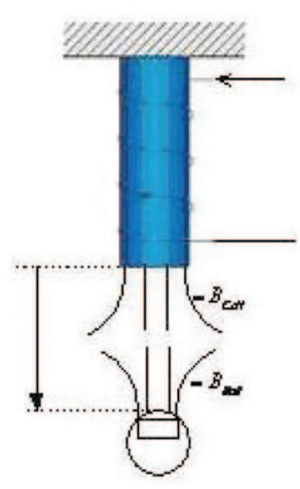

(b) Considered structure

Fig. 1. Principles of conventional and considered levitation systems

The principle of electromagnetic levitation can be illustrated by a simple one-dimensional system as shown in Fig.1 (a). By controlling the electric current flowing through coils around a solenoid, a conductible object, e.g., an iron or a steel ball, can be possibly levitated by the generated magnetic force. However, to develop a reliable and efficient levitation system is far from easy with respect to the fact that this kind of system is featured by 
complexity, nonlinearities, natural instability and large electromagnetic uncertainties (Gentili \& Marconi (2003); Kim (1997); Thompson (2000); Varella et al. (2004)).

A planar levitation system was investigated in (Kim (1997); Kim et al. (1998); Thompson (2000)). By conducting AC current through a disk-shaped insulated coil, the coil can be liftoff above a wide aluminum plate. The realized system is stable but under-damped without feedback control. The thermal problem is also investigated in (Thompson (2000)), and it turned out that the coil resistance increased significantly with the increase of temperature, which means that the system required more power in order to keep the levitated object at the same height when the temperature increases. As a consequence, the test setup can only be run for a few second at a time (Thompson (2000)). In order to control the levitated object's position and overcome the under-damping issue, a feedback mechanism is often required. The feedback control of a laboratory-sized one-dimensional levitation system is discussed in (Wong (1986)), and an analog lead compensator was developed using standard frequency response methods. Some application of advanced control methods such as the robust control and integrator back- stepping for magnetic bearing control can be found in (CST (1996)) and references therein. As we observed that most existing controllers are designed based on some kind of linear/linearized models and therefore linear. Measurements of the levitated object position and the current through the coil are often required by these controllers.

By focusing on the one-dimensional levitation, the comparison of system performances under a linear controller and a nonlinear controller was investigated in (Barie \& Chiasson (1996)). The nonlinear controller was developed by using feedback linearization based on a nonlinear model (Isidori (1989)). It showed that both controllers resulted more or less same system performances in terms of tracking capability for step-type references. However, the nonlinear controller is more sensitive to quantization error (e.g., 8 bit or 12 bit A/D convertors) in the current measurement. Regarding the sinusoid-type references, it turned out that the nonlinear controller resulted much better tracking performance than the linear controller did. However, the development of nonlinear controller heavily depends on the precision of available mathematical model. From practice point of view, no matter what kind of controller will be used, the thermal dynamic (heating coil) is always a critical concerning issue (Sønderskov \& Østerö (2007); Thompson (2000); Yang \& Pedersen (2006); Yang et al. (2007)).

Different from most existing one-dimensional levitation systems which use a conductible ball or coil as the levitated object (Barie \& Chiasson (1996); Gentili \& Marconi (2003); Oliveira et al. (1999); Wong (1986); Yang \& Pedersen (2006), here we consider a onedimensional levitation system with a permanent magnet object instead, i.e., a small NIB (Neodymium, Iron, Boron) magnet is glued at the inside top of a plastic ball as shown in Fig.0 (b). The main benefits of this configuration lie in the following perspectives:

- The solenoid's overheating problem is moderated. It is known that a large magnetic field is often required to levite a conductible object even with a relatively small operating range. It means that the coils must provide a large amount of current which directly leads to the heat dissipation problem (Thompson (2000)). Instead of purely depending on the coils, the magnetic field generated in the proposed configuration consists of contributions from the permanent NIB magnet as well as the contribution from coils around the solenoid.

- The system's operating range is enlarged under the same solenoid condition compared with the standard configuration (with conductible object). The magnetic field is 
considerably enhanced due to the contribution from the NIB magnet. In our constructed system the NIB contributes 4-5 times more flux density than the solenoid operating at the maximal current (Sønderskov \& Østerö (2007); Yang et al. (2007)).

However, the payoff of the above benefits is the complexity. The proposed configuration makes modeling and control of this kind of levitation system much more complicated regarding the fact that a permanent magnet is attached on a moving object (Simpson (1999)). This paper will explore the modeling, control and implementation of the proposed levitation system. First of all, the magnetic field generated by the moving NIB is experimentally investigated and modeled. Then a nonlinear model of the entire system is derived. System parameters are identified using some experimental ways. Afterwards a set of PID controllers are designed via trial-and-error method and automatic tuning using genetic algorithms, respectively. The developed controllers are implemented in the PC-supported LabView environment. The experimental tests show some good system performances. The rest of the paper is organized as: Section 2 gives a brief description of our benchmark system; Section 3 derives the nonlinear model of the considered system and then identifies the system coefficients by experiments; Section 4 analyzes the PID control design, automatic tuning and implementation issues; Section 5 discusses experimental results and we conclude the paper in Section 6.

\section{Experimental apparatus}

A one-dimensional levitation system is constructed using an aluminium framework as shown in Fig. 2. The electromagnet device consists of a solenoid with an iron core which is composed of thin steel plates riveted together. The levitated object is a plastic ball with diameter of $2 \mathrm{~cm}$. There is a small NIB magnet glued to the top inside the ball, and a M4 nut glued to the bottom acting as the counterweight to the NIB magnet. On the sides of the framework, slits are milled for ease of mounting and adjustment of the optical sensor system.

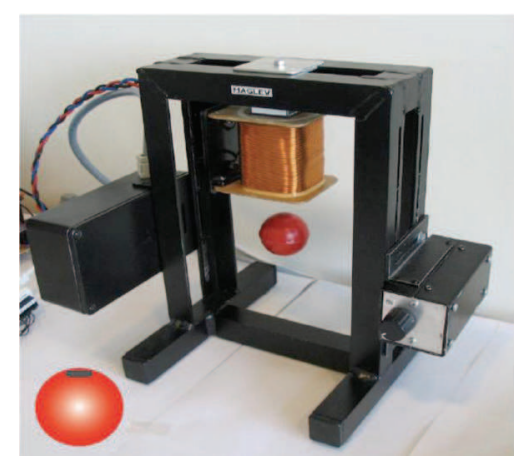

Fig. 2. Experimental laboratory setup

\subsection{Position sensor}

An optical sensor system for measuring the distance between the solenoid bottom and the levitated ball is developed using two LEDs (IR333-A) and a photodiode array (Hamamatsu 16- element Si photodiode array, type S5668-1). The sensor system is mounted inside an 
aluminium house with a milled slit facing to the possible operating range. As shown in Fig. 3., when the ball enters the detectable area, it casts a shadow on the photodiode array which leads to changes of currents. By measuring these currents, the position can be estimated by $x=\frac{L}{2} \frac{I_{2}-I_{1}}{I_{2}+I_{1}}$ where $I_{1}$ and $I_{2}$ are the currents through the photodiodes as shown in Fig. 3. $x$ is the upper boundary of the shadow on the position sensor, and $L$ is the length of the detectable area, which is $6 \mathrm{~mm}$ in our case. The measured current is converted to a voltage through the diagram as shown in Fig. 4.
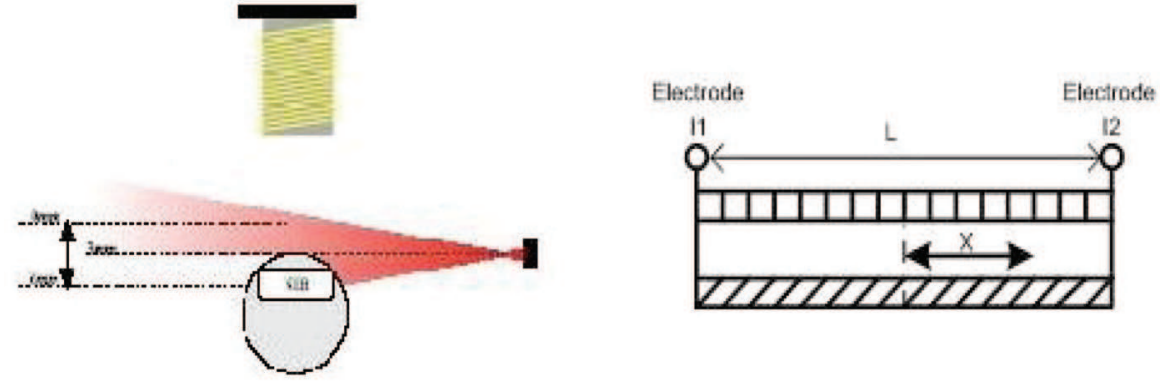

Fig. 3. Principle of the position detection

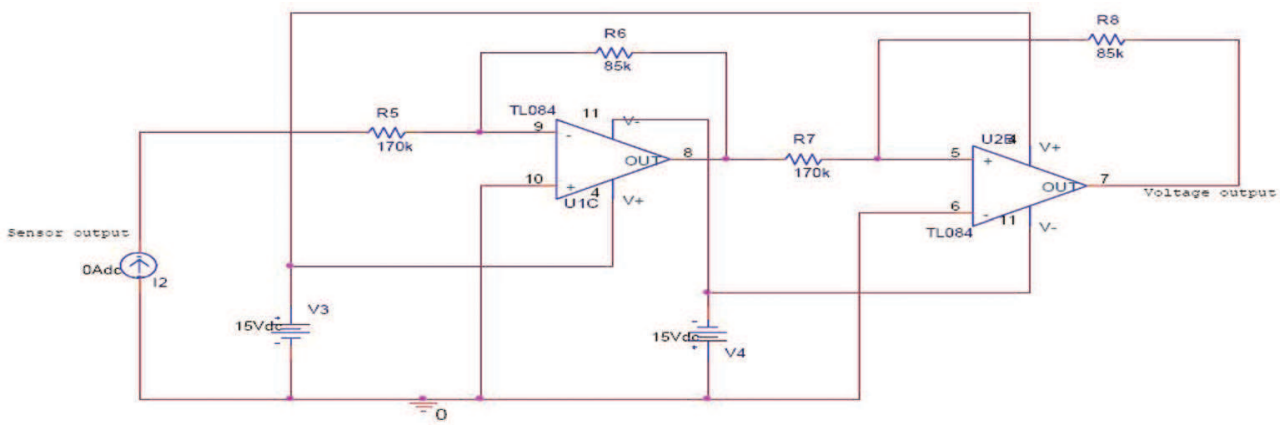

Fig. 4. Current-to-voltage conversion of the sensor measurement

\subsection{Current generator}

The current control scheme (Yang \& Pedersen (2006)) is employed for the control purpose instead of the conventional voltage control (Barie \& Chiasson (1996); Oliveira et al. (1999); Wong (1986)), such that the current drifting problem due to the thermal dynamic of the solenoid can be avoided. The basic scheme of the proposed current control is shown in Fig. 5. A digital- to-analog converter named AD7523 (Intersil) is used to converter the digital control signal into a analog voltage signal with a span of $200 \mathrm{mV}$. Through the opamp U3B (TL082) a new voltage signal with a span of $5 \mathrm{~V}$ is generated and used to control the open and close of the MOSFET transistor IRFZ44. In order to protect the MOSFET transistor IRFZ44 from the high voltage peaks, a varistor S14K30AUTO is placed between the drain and ground (Sønderskov \& Østerö (2007)). 


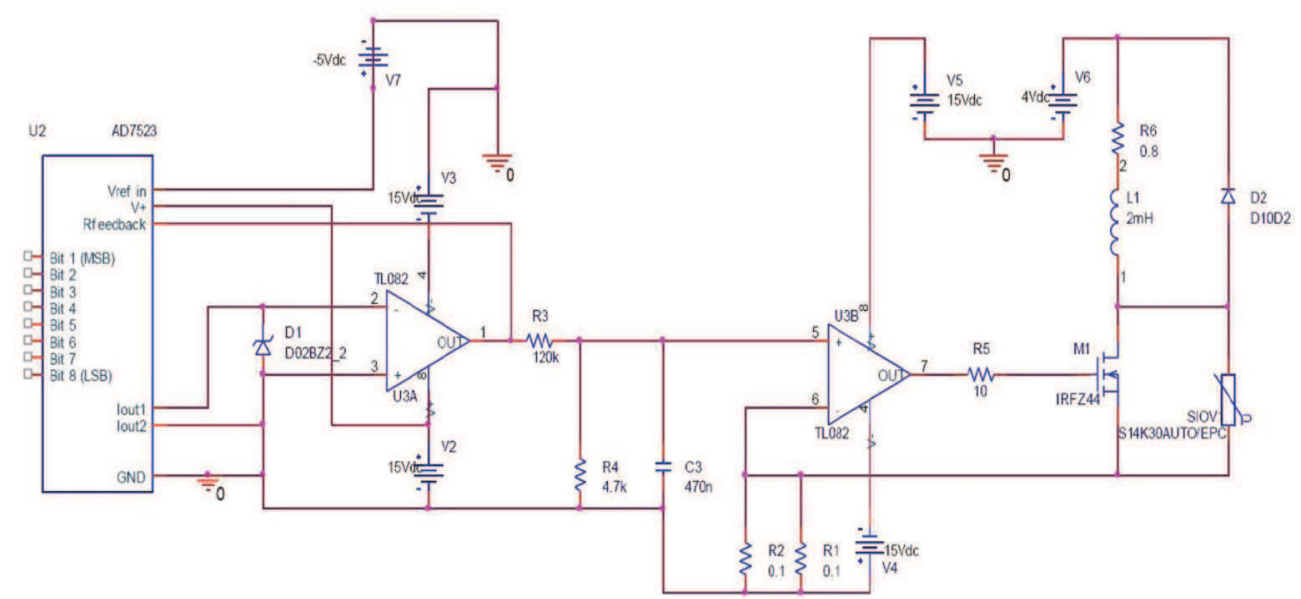

Fig. 5. Diagram of the current generator

\subsection{LabView environment}

The control algorithm is implemented in the National Instruments (NI) LabView environment for Windows XP. A Data Acquisition (DAQ) card typed NI PCI 6229 is used as the interface between the physical hardware and the LabView software. More information can be found in (Sønderskov \& Østerö (2007)).

\section{Modeling and identification}

The entire magnetic field in the considered setup consists of two distinguished parts: contribution from the permanent NIB magnet attached on the ball, and contribution from the solenoid when electric current flows through it. This magnetic field can be expressed as

$$
\vec{B}_{t}=\vec{B}_{c}+\vec{B}_{b}
$$

where $\vec{B}_{t}$ is the total magnetic field, $\vec{B}_{c}$ is the magnetic field induced by the solenoid, and $\vec{B}_{b}$ is the field induced by the NIB magnet. In the following, the feature of $\vec{B}_{b}$ is first investigated based on the setup. Then the total field $\vec{B}_{t}$ is analyzed using a theoretical approach. System parameters are identified through experiments.

\subsection{NIB magnetic field $\vec{B}_{b}$}

The NIB magnetic field is investigated through an experiment way. It is obvious that the magnetic field $\vec{B}_{b}$ will be influenced if the distance between the solenoid and the ball becomes smaller even without any current running in the coils around the solenoid (Woodson \& Melche (1968)). Thereby we define the magnetic field generated by the NIB magnet as a function of the distance between the bottom of the solenoid and the top of the ball, denoted as $\vec{B}_{b}(x)$, where $x$ is the mentioned distance. This magnetic field function can 
be measured by attaching a Hall Effect sensor at the top of the ball and manually moving the ball up or down within the possible working range. One measurement is shown in Fig. 6. By using the curve fitting technique, a 4th order polynominal is obtained as

$$
B_{b}(x)=a_{4} x^{4}+a_{3} x^{3}+a_{2} x^{2}+a_{1} x+a_{0},
$$

with coefficients listed in Table. 1. In the following, equation (2) is used as the model of NIB magnetic field.

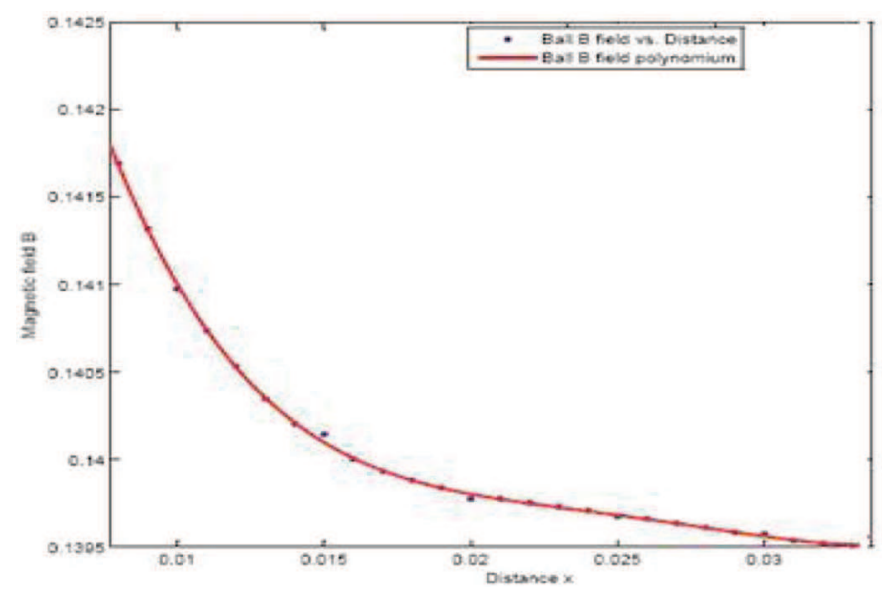

Fig. 6. Measured magnetic field generated by NIB via distance

\begin{tabular}{|c|c|}
\hline$a_{0}$ & 0.1417 \\
\hline$a_{1}$ & $-0.1196 \times 10^{-1}$ \\
\hline$a_{2}$ & $0.6905 \times 10^{2}$ \\
\hline$a_{3}$ & $-0.1794 \times 10^{4}$ \\
\hline$a_{4}$ & $0.1734 \times 10^{5}$ \\
\hline
\end{tabular}

Table.1. Coefficients of $B_{b}(x)$ induced by NIB magnet

\subsection{Nonlinear system model}

Under assumption that the used material has a linear characteristic, i.e., the magnetization density only depends on the magnetic field density (Woodson \& Melcher (1968)), the magnetic flux of the entire field, denoted as $\lambda(t)$, can be approximated by

$$
\lambda(t) \hat{=} L(x(t)) i(t)+\lambda_{B}(x(t)),
$$

where $i(t)$ denotes the current through the solenoid, and $x(t)$ denotes the displacement of the levitated object to the solenoid bottom. $L(x)$ denotes the inductance when the levitated object is assumed to be iron/steel and it can be regarded as a function of $x(t)$ (Wong (1986); Yang \& Pedersen (2006)). $\lambda_{B}(x)$ is the flux introduced by the NIB magnet, and it also is a 
function of $x(t)$ as we find out in eq.(2). By using the proposed approximation in (Wong (1986); Oliveira et al. (1999); Yang \& Pedersen (2006)), L(x) can be expressed as

$$
L(x)=L_{1}+\frac{L_{0}}{1+\left(\frac{x}{a}\right)},
$$

where $L_{0}=L(0)-L(\infty), L_{1}=L(\infty)$ and $a$ is a constant coefficient.

According to the electromagnetic theory (Woodson \& Melcher (1968)), the magnetic coenergy, denoted as $W$, can be calculated as

$$
W(t)=\int_{0}^{i(t)} \lambda(\bar{i}, x(t)) d \bar{i} .
$$

By inserting (3) and (4) into (5), there is

$$
W(t)=\frac{1}{2}\left(L_{1}+\frac{L_{0}}{1+\left(\frac{x(t)}{a}\right)}\right) i^{2}(t)+\lambda_{B}(x(t)) i(t) .
$$

The introduced magnetic force, denoted as $f(t)$, is determined from the magnetic co-energy according to

$$
f(t)=\frac{\partial W(t)}{\partial x_{a}},
$$

where $x_{a}$ represents the force acting axis, which is equal to the $x(t)$ axis. Then from (6) we have

$$
f(t)=-\frac{1}{2} \frac{L_{0} i^{2}(t)}{a\left(1+\frac{x(t)}{a}\right)^{2}}+\frac{d \lambda_{B}(x(t))}{d x} i(t) .
$$

Assume that the magnetic flux $\lambda_{B}(x)$ and the magnetic flux density have a constant linear relationship. It could be reasonable if the considered system only has small moving distance. There is

$$
\lambda_{B}(x) \stackrel{=}{=} \beta_{B} B_{b}(x)
$$

where $\boldsymbol{B}_{b}(x)$ is the value calculated from equation (2). Therefore, $\frac{d \boldsymbol{\lambda}_{B}(x)}{d x}$ can be approximated by

$$
\frac{d \lambda_{B}(x)}{d x}=4 a_{4} x^{3}+3 a_{3} x^{2}+2 a_{2} x+a_{1},
$$


with coefficients given in Table 1 .

Denote the mass of the levitating object as $m$ and the gravity acceleration as $g$. By neglecting the air drag friction, the dynamic of the levitating object can be obtained from Newton's Second Law as

$$
m \frac{d^{2} x(t)}{d t^{2}}=m g+f(t)
$$

By inserting (7) into the above equation, there is

$$
m \frac{d^{2} x(t)}{d t^{2}}=m g-\frac{1}{2} \frac{L_{0} i^{2}(t)}{a\left(1+\frac{x(t)}{a}\right)^{2}}+\frac{d \lambda_{B}(x(t))}{d x} i(t) .
$$

Compared with models used in (Barie \& Chiasson (1996); Oliveira et al. (1999); Pedersen \& Yang (2006); Wong (1986); Yang \& Pedersen (2006)), the third term on the right side of equation (10) is new and it is due to the existing of the permanent NIB magnet.

Through circuit analysis, the electrical perspective of the solenoid can be modeled as

$$
u(t)=R i(t)+\frac{d(L(x(t)) i(t))}{d t}+\frac{d \lambda_{B}(x(t))}{d t}
$$

where $R$ is the coil resistance, and $u(t)$ is the input voltage to the coil. Compared with models used in (Barie \& Chiasson (1996); Oliveira et al. (1999); Pedersen \& Yang (2006); Wong (1986); Yang \& Pedersen (2006)), the second term on the right side of (11) is new, and it is the EMF induced by the permanent NIB magnet.

By taking relationship (8) and substituting (4) and (2) into (11), there is

$$
\begin{gathered}
\frac{d i(t)}{d t}=\frac{a R+R x(t)}{a L(0)+L_{1} x(t)} i(t)+\frac{a L_{0}}{(a+x(t))\left(a L(0)+L_{1} x(t)\right)} i(t) \frac{d x(t)}{d t} \\
+\frac{a+x(t)}{a L(0)+L_{1} x(t)} u(t) \\
-\frac{\beta_{B}(a+x(t))}{a L(0)+L_{1} x(t)}\left(4 a_{4} x^{3}(t)+3 a_{3} x^{2}(t)+2 a_{2} x(t)+a_{1}\right) \frac{d x(t)}{d t} .
\end{gathered}
$$

Without triviality, if a small operating range is considered, the inductance (4) can be approximated by a constant value $(L=0.1398 H)$. In addition, by taking the linear part of $\lambda_{B}(x)$, a linear version of equation (12) can be derived as

$$
u(t)=R i(t)+L \frac{d i(t)}{d t}+\beta_{B} a_{1} \frac{d x(t)}{d t}
$$

which is similar to a simplified linear DC-motor model (Woodson \& Melcher (1968)). Equations (10) and (12) constitute of a nonlinear model of the considered levitation system. 
Compared with models used in (Barie \& Chiasson (1996); Oliveira et al. (1999); Pedersen \& Yang (2006); Wong (1986); Yang \& Pedersen (2006)), here the influence of the NIB magnet is reflected by the extra force in (10) and the EMF part in (12), respectively.

\subsection{Coefficient identification}

System coefficients $L_{0}, L(0)$ and $L_{1}$ used in (4) can be directly measured or estimated. However, coefficients $a$ and $\beta_{B}$ in (10) need to be identified through an experimental approach similar to those used in (Oliveira et al. (1999); Yang \& Pedersen (2006)). A set of experiments is organized to find the currents required to levitate the object at different equilibrium positions. The result is plotted in Fig. 7.

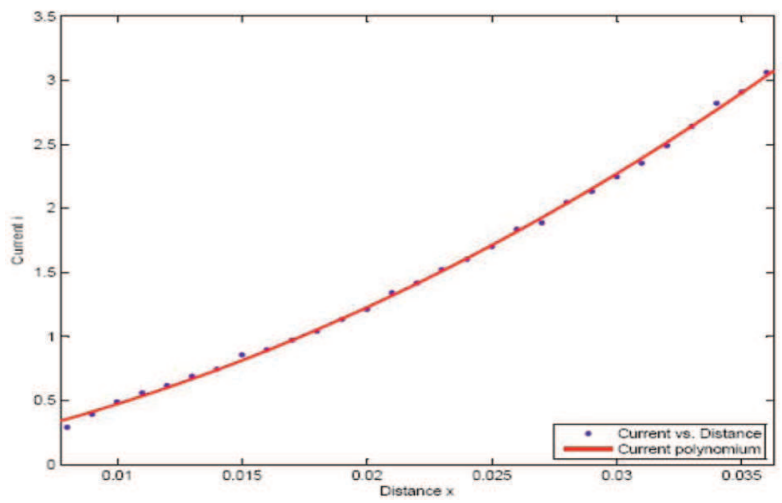

Fig. 7. The equilibrium points and corresponding required currents

By picking up three close equilibrium points and their corresponding currents, denoted as $x_{1}, x_{2}, x_{3}$ and $i_{1}, i_{2}, i_{3}$, respectively, from (7) there is

$$
\frac{1}{2} \frac{L_{0} i_{1}^{2}}{a\left(1+\frac{x_{1}}{a}\right)^{2}}-\beta_{B} B_{b}\left(x_{1}\right) i_{1}=\frac{1}{2} \frac{L_{0} i_{2}^{2}}{a\left(1+\frac{x_{2}}{a}\right)^{2}}-\beta_{B} B_{b}\left(x_{2}\right) i_{2},
$$

and

$$
\frac{1}{2} \frac{L_{0} i_{1}^{2}}{a\left(1+\frac{x_{1}}{a}\right)^{2}}-\beta_{B} B_{b}\left(x_{1}\right) i_{1}=\frac{1}{2} \frac{L_{0} i_{3}^{2}}{a\left(1+\frac{x_{3}}{a}\right)^{2}}-\beta_{B} B_{b}\left(x_{3}\right) i_{3} .
$$

Coefficient $a$ can be calculated by combining the above two equations. After $a$ is determined, $\beta_{B}$ can be determined based on any set, e.g., set $\left(x_{1}, i_{1}\right)$. A simple way to determine $a$ is to assume the term $\beta_{B} B_{b}\left(x_{k}\right) i_{k}$ is almost constant for $k=1,2,3$. This assumption is reasonable for a small operating range, so $a$ can be determined by

$$
a=\frac{i_{2} x_{1}-i_{1} x_{2}}{i_{1}-i_{2}},
$$

Correspondingly, $\beta_{B}$ can be determined by 


$$
\beta_{B}=\frac{m g+\frac{1}{2} \frac{L_{0} i_{1}^{2}}{a\left(1+\frac{x_{1}}{a}\right)^{2}}}{B_{b}\left(x_{1}\right) i_{1}} .
$$

Average values of obtained $a$ and $\beta_{B}$ and other directly measured coefficients are listed in the Table 2.

\begin{tabular}{|c|c|c|c|}
\hline Description & Symbol & Value & Unit \\
\hline Coil inductance & $L_{1}$ & $2.0 \times 10^{-3}$ & $\mathrm{H}$ \\
Coil resistance & $R$ & 0.8 & $\Omega$ \\
Inductance & $L_{0}$ & $0.99 \times 10^{-3}$ & $\mathrm{H}$ \\
Mag. ind. coeff. & $a$ & 1.18 & $\mathrm{~m}$ \\
Mass of object & $m$ & $4.27 \times 10^{-3}$ & $\mathrm{~kg}$ \\
Flux coeff. & $\beta$ & 0.065 & \\
\hline
\end{tabular}

Table 2. Parameters used in the considered system

Due to the considered levitation system being naturally unstable and having a very fast response, it is difficult to validate the developed model directly. Therefore, a simple PID feedback controller is developed to keep the considered system operating properly. The mathematical model is validated by comparing the simulated closed-loop control system and the real controlled system afterwards (Yang et al. (2007)).

\section{Design and implementation of PID controllers}

\subsection{Empirical PID controller}

By using the obtained nonlinear model, an analog PID controller is developed and manually tuned based on the Ziegler-Nichols PID tuning method. Then the developed PID controller is discretized with a sampling frequency of $480 \mathrm{~Hz}$, which is determined by the NI DAQ card used for the digital implementation. The implemented controller has the form

$$
\begin{aligned}
& u(k)=u(k-1)+K_{p}\left(1+\frac{T}{T_{i}}+\frac{T_{d}}{T}\right) e(k) \\
& -K_{p}\left(1+2 \frac{T_{d}}{T}\right) e(k-1)+K_{p} \frac{T_{d}}{T} e(k-2),
\end{aligned}
$$

where $T, K_{p}, T_{i}$ and $T_{d}$ are sampling period, P, I, and D coetcients, respectively. $e(k)$ is the displacement tracking error. The simulation of the closed-loop control system using the empirical PID controller is shown in Fig.8. It can be observed that the controlled system has a reasonable response time and good tracking capacity.

\subsection{Automatic tuning of PID controller using GA algorithms}

From our preliminary investigation (Pedersen \& Yang (2006); Yang \& Pedersen (2006)), it turned out that the PID controller can be automatically tuned using the multi-objective nondominated sorting genetic algorithm (NSGA-II) based on the nonlinear system model. 
The performance induced by different PID-controller parameters are evaluated by the following criteria based on the step response:

- Overshoot $\left(M_{p}\right)$;

- $\quad$ Rise time $\left(t_{r}\right)$;

- $\quad$ Settling time $\left(t_{s}\right)$; and

- Integrated absolute error (IAE).

An illustration of the performance measures is given in Fig. 9. Each of these performance measures will be included as objectives to be minimized as their inter-dependence will depend highly on the nonlinear system expressed by (10) and (12).

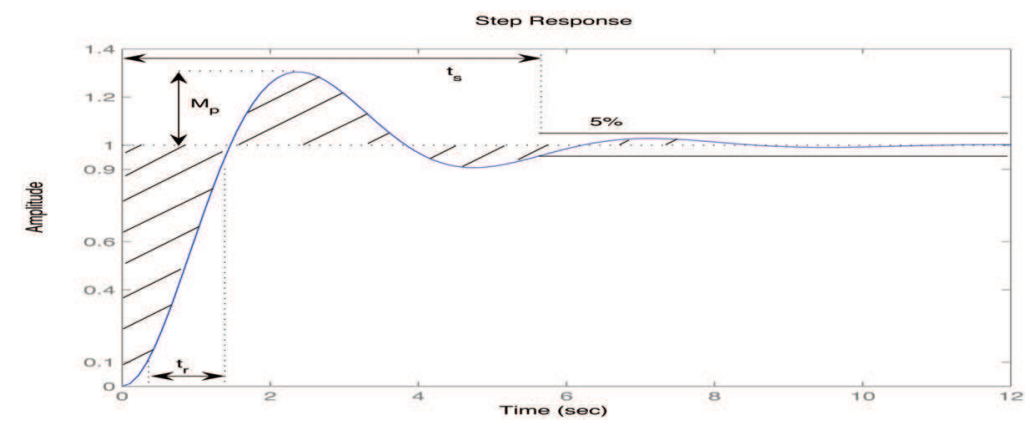

Fig. 9. Performance measures for step response

The non-dominated sorting genetic algorithm (NSGA-II) developed in (Deb et al. (2000)) is a multi-objective algorithm, which can evolve a set of non-dominated solutions that are all equally well suited for solving the specific problem given the performance measures specified. Many of the NSGA-II run-time parameters used for here are the same as the NSGA-II default values (Pedersen \& Yang (2006); Yang \& Pedersen (2006)), such as

\begin{tabular}{|c|c|}
\hline Description & setting \\
\hline Crossover probability & 0.9 \\
Mutation probability & 0.33 \\
SBX parameter & 10 \\
Mutation parameter & 50 \\
Rigid bounds & 1 (yes) \\
\hline
\end{tabular}

Table. 3. Parameters used for running NSGA-II

In the simulation, The range for $K_{p}$ is set to [-1000,0]. The ranges for $T_{i}$ and $T_{d}$ are both set to $[0,15]$. With respect to the computational complexity of the simulations, a population size of 50 individuals was chosen along with a maximum number of generations of 150 . Besides from the use of the 4 objectives a constraint on the allowable amount of overshoot has also been formulated as only values below $100 \%$ was allowed. The distribution of $K_{p}, T_{i}$ and $T_{d}$ for the case where the outliers have been removed is illustrated in Fig. 10.

It is quite obvious that there is a large grouping of individuals for small values of $T_{i}$ and $K_{p}$ values below -800 . A simulation of a typical controller from this cluster, with parameters as $K_{p}=-800.46, T_{i}=0.021$ and $T_{d}=0.06$, is shown in Fig. 11 .

The corresponding performance measures for this individual are IAE $=5 \cdot 10^{-4}, M_{p}=84.82 \%$, $t_{r}=21 \mathrm{~ms}$ and $t_{s}=0.425 \mathrm{~s}$. It can be observed that the system response consists of a fast 
oscillation on top of a slower one. The fast rise time is mainly due to the size of $K_{p}$ which is obviously very aggressive towards positional errors.

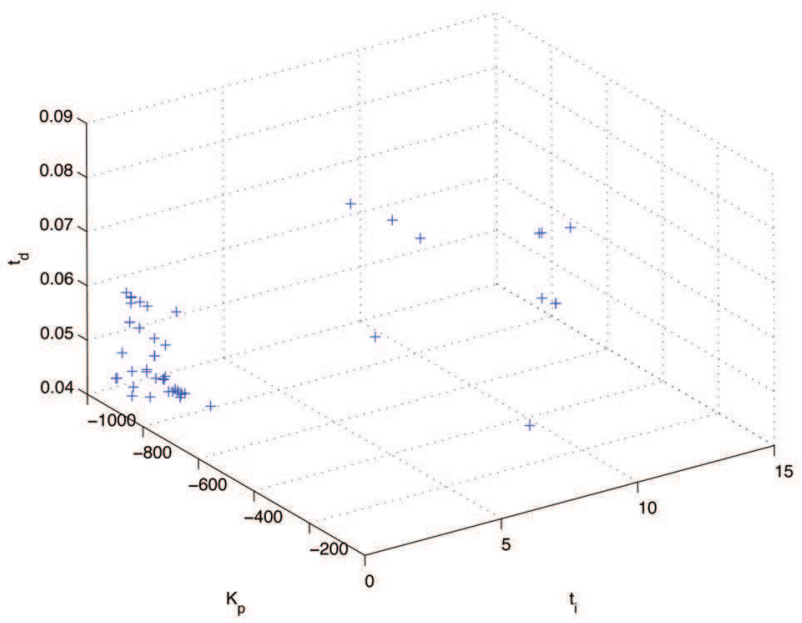

Fig. 10. Plot of parameters $K_{p}, T_{i}$ and $T_{d}$ for last generation
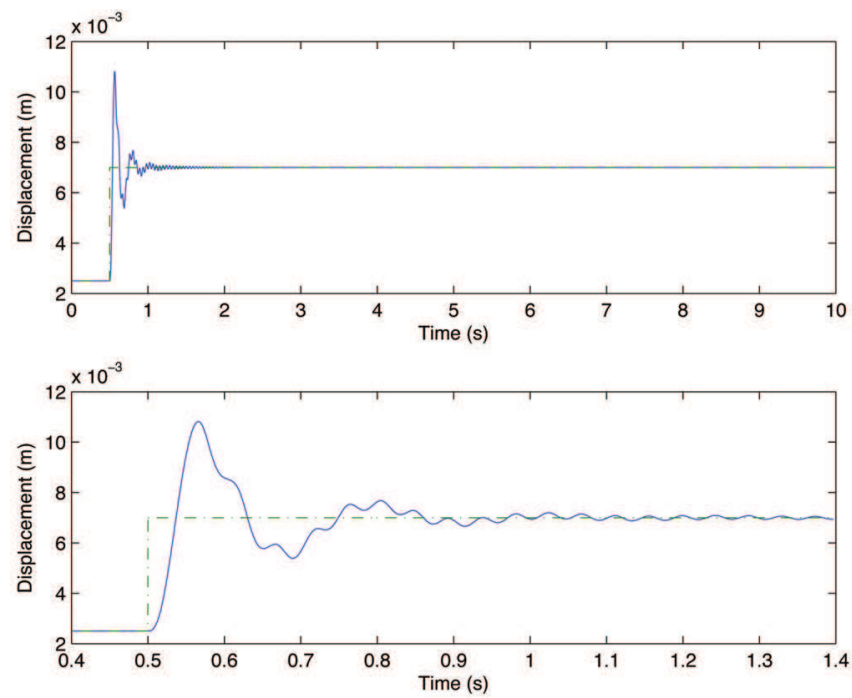

Fig. 11. System step response in simulation

\subsection{LabView Implementation}

The developed controllers are implemented in NI LabView environment on a PC running Windows XP. Therefore some attention needs to be paid on the real-time issues. For instance, the connection between the external devices and the LabView environment is setup manually, even though the DAQ assistant in LabView could more easily create the 
communication line. However, our experiences showed that the DAQ Assistant is quite time consuming, no matter if it is used inside or outside the timed loop (Sønderskov \& Østerö (2007); Yang et al. (2007)). Another real-time issue relevant to the Windows XP operating system. It is well known that Windows XP gives priority to different processes that are executed. For example, just moving the mouse is sometimes enough to slow down the execution of LabView code. In order to solve this real-time problem, the timed loop structure is used in the LabView program, which guarantees that the LabView code should be executed within the defined time period. Furthermore, In order to check the sampling rate issues, a sampling frequency calculator is constructed as shown in Fig. 12. A front panel of the developed controller is shown in Fig.13.

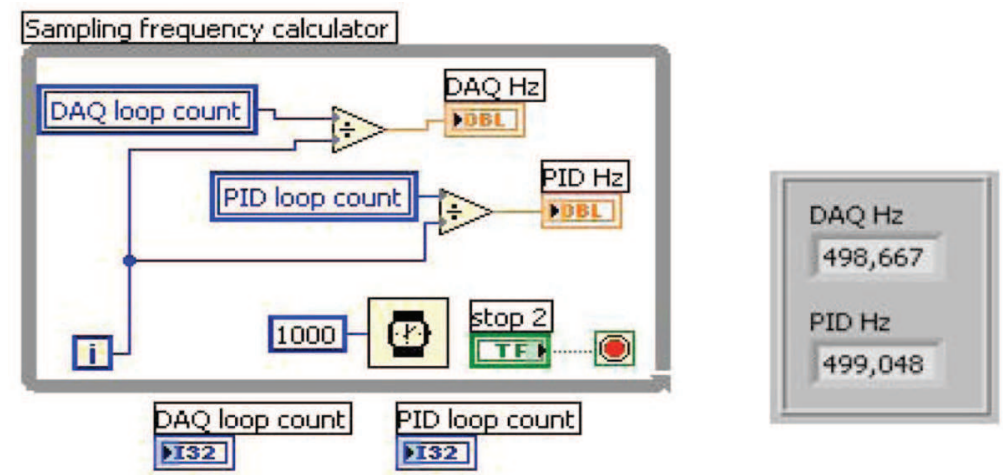

Fig. 12. Sampling frequency calculator with front panel indicators

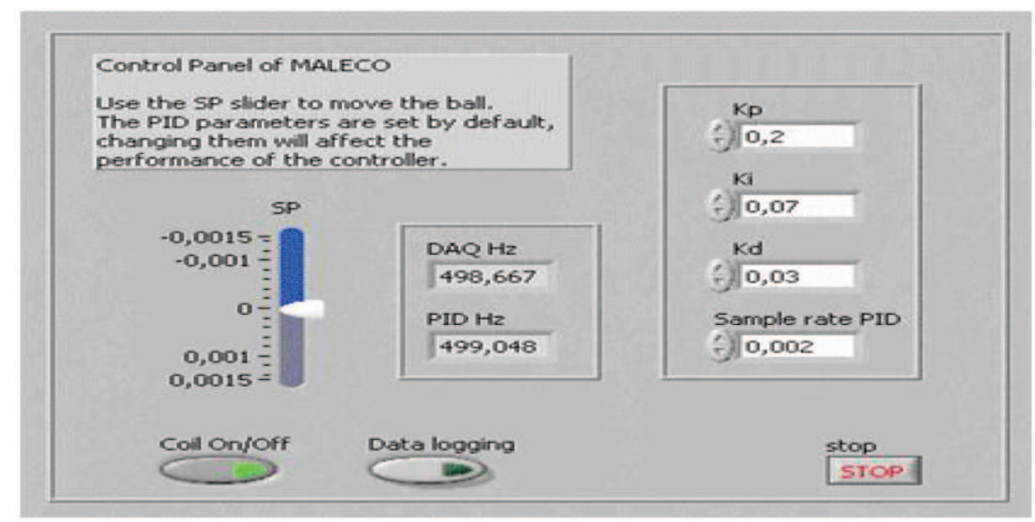

Fig. 13. Front panel of the developed controller

\section{Testing results and discussions}

The simulated performance of the closed-loop control system using the empirical PID controller is shown in Fig. 8. The same controller is implemented in the LabView program and tested with the physical setup. One test result based on the same set of set-points as for simulation is shown in Fig. 14. It can be observed that in principle the controlled physical 
system has quite similar performance as the simulation model. However, it is also obvious that the controlled physical system has much shorter response time and much larger overshot and oscillation compared with the simulated system performance. The reasons for these deviations could be explained in the following perspectives:

- Imprecise sensor measurement. The optical position sensor is very sensitive to light disturbances;

- Frequent switchings of the MOSFET IRFZ44. The frequent on-off switchings of current due to this MOSFET can directly lead to oscillations in real tests (Yang et al. (2007));

- Imprecise sampling rates of DAQ card and PID computation due to the real-time problem of Windows XP operating system. This could cause synchronization problems in data acquisition and control computation;

- the approximation of system coefficients. For example, in a strict sense, the system coefficient $\beta_{B}$ should be displacement dependent. However, we assume it is always constant due to simplicity.

The consistency between simulation and real tests could be improved if above problems could be solved or moderated. By softly changing the set-points, e.g., filtering the rectangular set- points, the controlled physical system shows a better performance as shown in Fig. 15. It can be observed that the large overshot that appeared in Fig. 14 has disappeared.

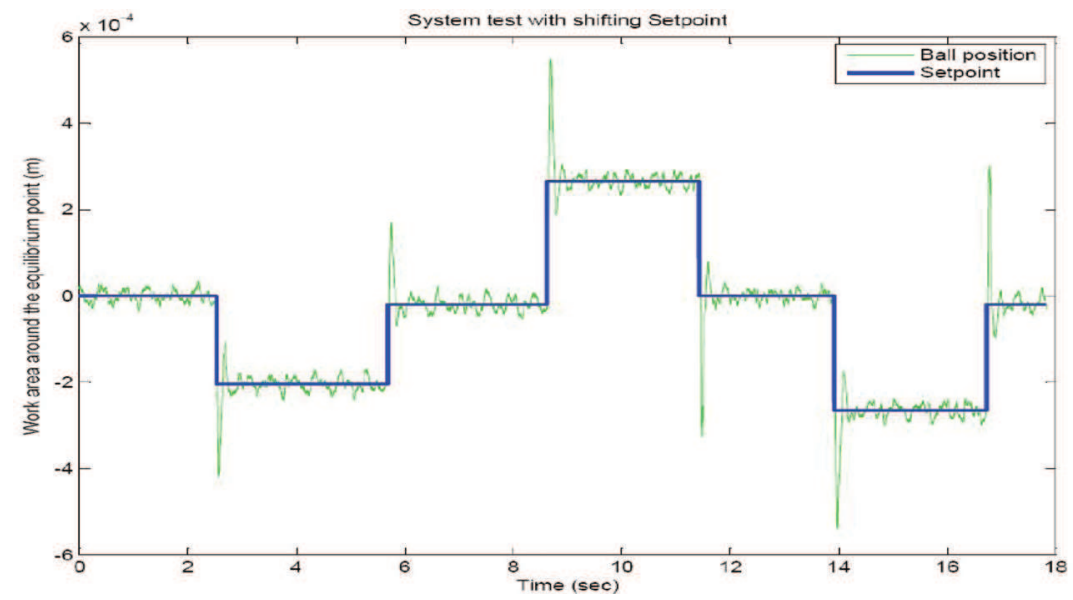

Fig. 14. Response of the controlled physical setup

One test result using the same control coefficients directly from NSGA-II tuning is shown in Fig. 16. Compared with the simulation result shown in Fig. 11, this implemented controller has quite similar behavior as simulation study. However, it is also obvious that the fast dynamic has much larger amplitude than it does in simulation, which could be due to the following facts:

- The designed closed-loop system is obviously under-damped;

- The influence from the external disturbances, e.g., the air flow around the ball etc;

- Model uncertainties and unprecise position measurements.

More analysis of these issues will be one part of our future work. 


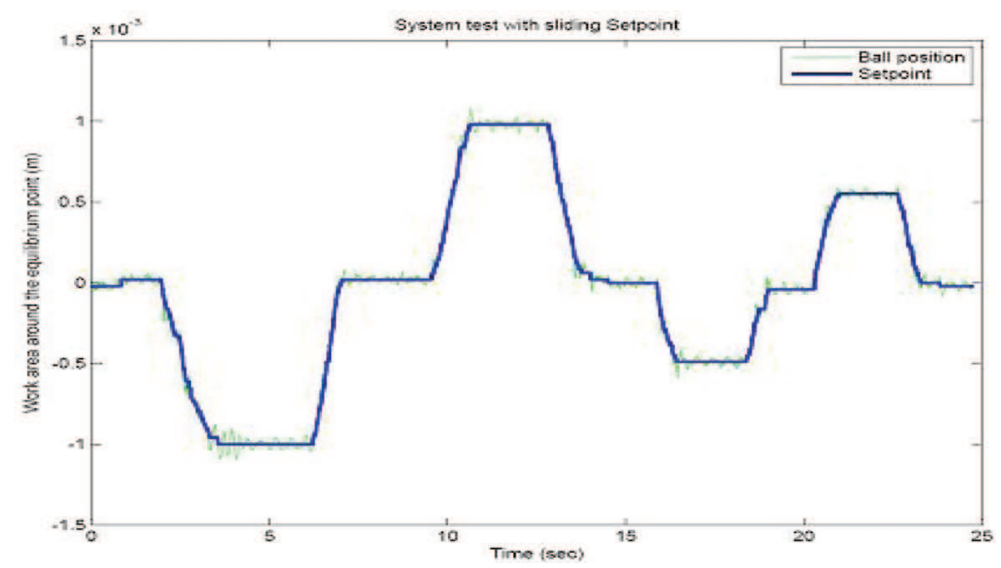

Fig. 15. Response of the controlled physical setup with soft changes

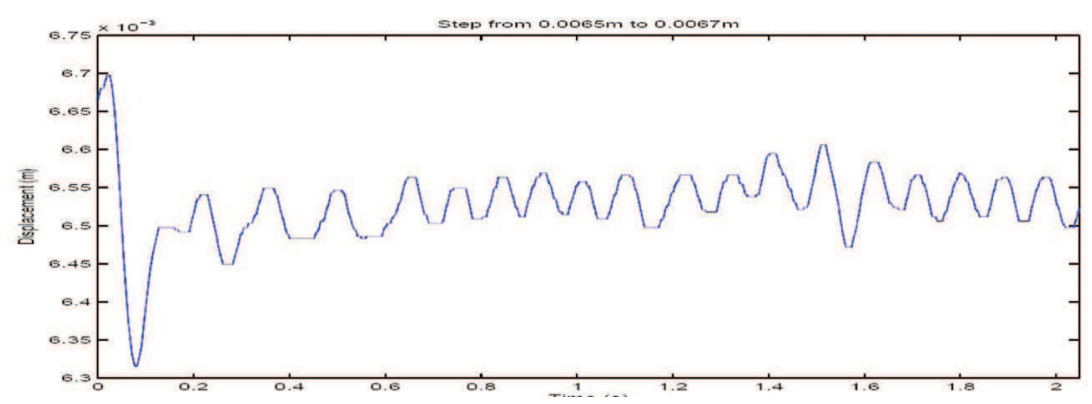

Fig. 16. Step response of the controlled setup using the NSGA-II tuned controller

\section{Conclusion}

The modeling and control of a 1-D magnetic levitation system with a permanent magnet object is investigated. The feature of the moving permanent magnet is explored using an experimental method and it is modeled through curve fitting technique. The entire system model is derived based on the electromagnetic theory and afterward system coefficients are identified through designed experiments. The developed model is validated through performance comparison of the closed-loop model and the controlled physical system.

The PID control is chosen as the control structure at this stage regarding the fact: (1) it is simple and require few computation resources; (2) The developed PID controllers only need the position information, with no need for the current measurement and speed estimation, such that the potential degradation of the system performance due to quantization (Barie \& Chiasson (1996)) can be minimized;

The developed controllers are implemented in the LabView environment based on a PC running Windows XP. The real-time issues are managed by additional programs. Both simulation and real tests showed a clear consistency and a good system performance. Furthermore, The investigation of using genetic algorithms to automatically tune PID controller shows a potential to use this artificial intelligence method for supporting the control design for complicated nonlinear systems. 


\section{Acknowledgement}

The authors would like to thank René M. Sønderskov, Kim S. Østerö, Niels A. Pedersen, Stefan K. Greisen and Jette R. Hansen for their contributions in system development and laboratory tests.

\section{References}

Special issue on magnetic bearing control. IEEE Control Systems Technology, Sept 1996.

W. Barie and J. Chiasson. Linear and nonliear state-space controllers for magnetic levitation. Int. J. of Systems Science, 27(11):1153-1163, 1996.

K. Deb, A. Pratap, and S. Moitra. A fast elitist non-dominated sorting genetic algorithm for multi-objective optimization: Nsga-ii. Parrallel Problem Solving from Nature PPSN VI, pages 849-858, 2000. NSGA-II code available at KanGAL website: http://www.iitk.ac.in/kangal.

L. Gentili and L. Marconi. Robust nonlinear disturbance suppression of a magnetic levitation system. Automatica, (39):735-742, 2003.

A. Isidori. Nonlinear Control Systems. New York: Springer-Verlag, 1989.

W. Kim. High-Precision Planar Magnetic Levitation. Phd thesis, Massachusetts Institute of Technology, June 1997.

W. Kim, D.L. Trumper, and J.H. Lang. Modeling and vector control of planar magnetic levitator. IEEE Trans. on Industry Applications, 34(6):1254-1262, Nov/ Dec 1998.

V.A. Oliveira, E.F. Costa, and J.B. Vargas. Digital implementation of a megnetic suspension control system for laboratory experiments. IEEE Trans. on Education, 42(4):315-322, Nov. 1999.

G.K.M. Pedersen and Z. Yang. Multi-objective pid-controller tuning for a magnetic levitation system using nsga-ii. In Maarten Keijzer, editor, Proceedings of Genetic and Evolutionary Computation Conference - GECC0 2006, pages 1737-1744, Seattle, Washington, USA, Jul 2006. ACM.

T.L. Simpson. Effect of a conducting shield on the inductance of an air-core solenoid. IEEE Trans. on Magnetics, 35(1):508-515, Jan 1999.

R.M. Sønderskov and K.S. Østerö. Malecos: Magnetic levitation control systems. 7th semester project report, Aalborg University Esbjerg, Denmark, Jan 2007.

M.T. Thompson. Electrodynamic magnetic suspension - models, scaling laws, and experimental results. IEEE Trans. on Education, 43(3):336-342, Aug. 2000.

M. Varella, E. Calloni, L.Di Fiore, L. Milano, and N. Arnaud. Feasibility of a magnetic suspension for second generation gravitational wave interferometers. Astroparticle Physics, (21):325-335, 2004.

R. Wisniewski and J. Stoustrup. Periodic h-2 synthesis for spacecraft attitude control with magnetorquers. Journal of Guidance Control and Dynamics, 27(5):874-881, 2004.

T.H. Wong. Design of a magnetic levitation control system - an undergraduate project. IEEE Trans. on Education, E-29(4):196-200, Nov 1986.

H. Woodson and J. Melcher. Electromechanical Dynamics - part I: Discrete Systems. Wiley, New York, 1968.

Z. Yang and G.K.M. Pedersen. Automatic tuning of pid controller for a 1-d levitation system using a genetic algorithm: a real case study. In Proceedings of the 2006 IEEE International Symposium on Intelligent Control, pages 3098-3103, Munich, Germany, Oct 2006. IEEE.

Z. Yang, G.K.M. Pedersen, and J.H. Pedersen. Modeling and control of one-dimensional magnetic levitation system with a permanent-magnet object. In Proceedings of the $13^{\text {th }}$ IEEE/IFAC International Conference on Methods and Models in Automation and Robotics, pages 723-729, Szczecin, Poland, Aug 2007. IEEE. 


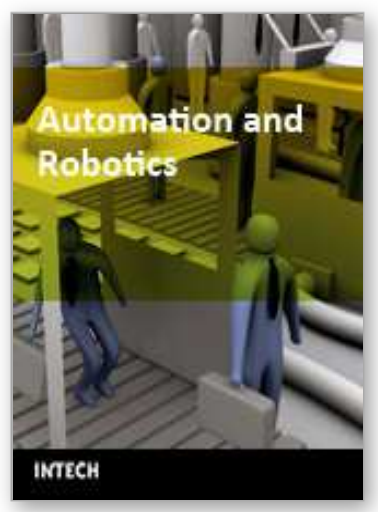

\author{
Automation and Robotics \\ Edited by Juan Manuel Ramos Arreguin
}

ISBN 978-3-902613-41-7

Hard cover, 388 pages

Publisher I-Tech Education and Publishing

Published online 01, May, 2008

Published in print edition May, 2008

In this book, a set of relevant, updated and selected papers in the field of automation and robotics are presented. These papers describe projects where topics of artificial intelligence, modeling and simulation process, target tracking algorithms, kinematic constraints of the closed loops, non-linear control, are used in advanced and recent research.

\title{
How to reference
}

In order to correctly reference this scholarly work, feel free to copy and paste the following:

Zhenyu Yang, Gerulf K.M. Pedersen and Jorgen H. Pedersen (2008). Model-Based Control of a Nonlinear One Dimensional Magnetic Levitation with a Permanent-Magnet Object, Automation and Robotics, Juan Manuel Ramos Arreguin (Ed.), ISBN: 978-3-902613-41-7, InTech, Available from:

http://www.intechopen.com/books/automation_and_robotics/modelbased_control_of_a_nonlinear_one_dimensional_magnetic_levitation_with_a_permanent-magnet_obje

\section{INTECH}

open science | open minds

\section{InTech Europe}

University Campus STeP Ri

Slavka Krautzeka 83/A

51000 Rijeka, Croatia

Phone: +385 (51) 770447

Fax: +385 (51) 686166

www.intechopen.com

\section{InTech China}

Unit 405, Office Block, Hotel Equatorial Shanghai

No.65, Yan An Road (West), Shanghai, 200040, China

中国上海市延安西路65号上海国际贵都大饭店办公楼 405 单元

Phone: +86-21-62489820

Fax: +86-21-62489821 
(C) 2008 The Author(s). Licensee IntechOpen. This chapter is distributed under the terms of the Creative Commons Attribution-NonCommercialShareAlike-3.0 License, which permits use, distribution and reproduction for non-commercial purposes, provided the original is properly cited and derivative works building on this content are distributed under the same license. 